\title{
Embedded System for Prosthetic Control Using Implanted Neuromuscular Interfaces Accessed Via an Osseointegrated Implant
}

\author{
Enzo Mastinu, Student Member, IEEE, Pascal Doguet, Member, IEEE, Yohan Botquin, Bo Håkansson, \\ and Max Ortiz-Catalan, Member, IEEE,
}

\begin{abstract}
Despite the technological progress in robotics achieved in the last decades, prosthetic limbs still lack functionality, reliability, and comfort. Recently, an implanted neuromusculoskeletal interface built upon osseointegration was developed and tested in humans, namely the Osseointegrated Human-Machine Gateway. Here we present an embedded system to exploit the advantages of this technology. Our Artificial Limb Controller allows for bioelectric signals acquisition, processing, decoding of motor intent, prosthetic control, and sensory feedback. It includes a neurostimulator to provide direct neural feedback based on sensory information. The system was validated using real-time tasks characterization, power consumption evaluation, and myoelectric pattern recognition performance. Functionality was proven in a first pilot patient from whom results of daily usage were obtained. The system was designed to be reliably used in activities of daily living, as well as a research platform to monitor prosthesis usage and training, machine learning based control algorithms, and neural stimulation paradigms.
\end{abstract}

Index Terms-Electromyography (EMG), prosthetic controller, osseointegration, pattern recognition, Osseointegrated HumanMachine Gateway (OHMG), sensory feedback.

\section{ACRONYMS}

$\begin{array}{ll}\text { AFE } & \text { Analog Front-End } \\ \text { ALC } & \text { Artificial Limb Controller } \\ \text { DC } & \text { Direct Control } \\ \text { EMG } & \text { Electromyography } \\ \text { ENG } & \text { Electroneurography } \\ \text { LDA } & \text { Linear Discriminant Analysis } \\ \text { MCU } & \text { Microcontroller Unit } \\ \text { MPR } & \text { Myoelectric Pattern Recognition } \\ \text { MSPU } & \text { Mixed Signal Processing Unit } \\ \text { NS } & \text { Neurostimulator } \\ \text { OHMG } & \text { Osseointegrated Human-Machine Gateway } \\ \text { PCCU } & \text { Prosthetic Control and Communication Unit } \\ \text { SVM } & \text { Support Vector Machine }\end{array}$

Research supported by Swedish Research Council (Vetenskapsrdet), VINNOVA and partially from EU project DeTOP.

E. Mastinu and B. Håkansson are with the Dept. of Electrical Engineering, Chalmers University of Technology, Gothenburg, Sweden (e-mail: enzo@chalmers.se, boh@chalmers.se).

P. Doguet and Yohan Botquin are with Synergia Medical, MontSaint Guibert, Belgium (e-mail: pascal.doguet@synergiam.com, yohan.botquin@synergiam.com).

M. Ortiz-Catalan is with the Dept. of Electrical Engineering, Chalmers University of Technology, and Integrum AB, Gothenburg, Sweden (e-mail: maxo@chalmers.se).

\section{INTRODUCTION}

D ESPITE the advances in prosthetic hardware that allow an increasing number of artificial joints to approach those of the lost limb [1], a major issue remains unsolved, namely, how to achieve a reliable and natural control of the prosthetic limb. After many years of research and development on prosthetics, amputees mostly rely on direct control (DC) (also known as one-for-one control, or one-muscle to one-function), which is often combined with sequential solutions for grasp switching based on encoding unnatural muscular activation (e.g., co-contraction) [2]. This control mechanism is pervasive owing to its simplicity, relative reliability, and ease to learn. Unfortunately, the functional outcome is commonly related to the specific patient predisposition, thus often resulting in rejection of the myoelectric prosthesis, or in reduction of the robotic potential to a simple prosthesis claw [3]. Modern prostheses are hindered by discomfort and poor functionality [4-6]. The latter has pushed research towards the challenge of using information from the neuromuscular system in more surgically and technologically sophisticated manners [7-9].

Comfort and functionality had been considerably improved by the use of osseointegration for direct skeletal attachment of limb prostheses [10], [11]. Osseointegration provides a longterm, mechanically stable interface between biology and the artificial limb, in which a titanium implant is surgically inserted into the remaining bone of the amputated extremity [11]. The osseointegration technology has been recently enhanced to allow bidirectional communication between implanted neuromuscular electrodes and the artificial limb [12]. This Osseointegrated Human-Machine Gateway (OHMG) combines the benefits of skeletal attachment with the reliability and increased information provided by implanted muscular electrodes [13], [14]. The first attempts on using implanted electrodes to restore sensory feedback were conducted over 40 years ago [15], and several others were reported more recently [16-19]. Despite the efforts, close-loop control has not been achieved yet in activities of the daily living arguably due to the lack of a suitable communication interface. The OHMG now provides a clinically viable long-term access to implanted neural interfaces that can be used for bidirectional communication to accomplish such purpose.

Here we present the development of an embedded system to exploit the advantages of the OHMG technology. Efforts were placed in hardware and software design, carefully seeking a 
balance between the computational demand and capabilities of the core processor. Our Artificial Limb Controller (ALC) was designed as a self-contained wearable unit capable of decoding motor volition and providing direct neural sensory feedback (Fig. 1). This ensemble provides a clinically viable solution for the control of upper limb prostheses, as well as a research platform for further investigations. The ALC's design and validation in real-time myoelectric pattern recognition (MPR) are described in this study.

\section{BACKGROUND AND RELATED WORK}

Prostheses are probably the first form of rehabilitation in history. In 1963 Wirta et al. reported a historical first example of an embedded MPR system [2]. A decade later similar technology was developed in Sweden [20]. In both studies, custom robotic arms were designed and Discriminant Analysis was chosen as the classification algorithm.

The interest on myoelectric control systems grew for decades developing in parallel with the computation capabilities of processing units. Most recent studies report similar approaches for real-time motor volition decoding using personal computers (PC) [8], [21].

In 2008, Tenore et al. demonstrated the challenges of implementing the MPR chain in a microcontroller (MCU) based system [22]. They trained their classifier using a PC and then used a fixed-point digital signal processor to compute floatingpoint operations. The additional efforts to compute floatingpoint calculations resulted in delays and reduced accuracy due to rescaling. They concluded that a processor natively capable of floating-point operations was necessary. Hirata et al. designed an embedded MPR system that allowed for onboard training [23], and a similar but open and configurable embedded platform for research purposes was developed by Liu et al. [24]. The previous studies relied on powerful but power demanding processors (e.g., PXA270-Intel and CortexA8-ARM), which poses energy challenges for a wearable solution. Recently, a low power solution was presented by Benatti et al. using an embedded controller based on a CortexM4 MCU with floating-point capabilities [25]. A Support Vector Machine (SVM) classifier was implemented using off-board training and reported a classification accuracy of $90 \%$. This accuracy was computed by an equivalent algorithm implemented in Matlab (Mathworks, USA) using a PC, thus limited information was provided on the MCU performance. All the aforementioned studies provided no assessment of realtime performance.

Approaches using field-programmable gate arrays (FPGA) have shown to be highly beneficial for accelerating the computation of pattern recognition algorithms [26], [27]. FPGAs represent a valuable solution for prosthetic control which is predestined to appear in future embedded MPR systems, but currently no clinical implementation of such system has been reported.

To date there is a single commercially available embedded MPR system from which limited information is available due to its commercial nature (COAPT, Chicago, USA). Although clinical investigations are ongoing, no results have been made publicly available in the scientific literature.
Here we present a low-power embedded MPR system validated in real-time. Our ALC contains a commercially available analog front-end (AFE) and a low power 32-bit MCU with a floating-point embedded unit. It follows the literature standards on electromyography (EMG) acquisition [28] and advanced myoelectric control techniques [29]. Direct control as well as two machine learning algorithms were implemented, namely Linear Discriminant Analysis (LDA) and SVM. All processing tasks were timed and a full report is provided here to support ALC real-time capabilities.

Sensory feedback plays an important role in human motor control, and therefore a neurostimulation stage was also included in our self-contained ALC to exploit the direct neural connection provided by the OHMG [12]. The ALC was also instrumented with inertial sensors as this might further improve controllability by mitigating the effect of arm positioning [31], or simply to exploit sensor fusion approaches [32].

The ALC was designed to be a clinically viable solution for daily life use, as well as a research platform compatible with the OHMG. It allows for close-loop control of myoelectric prostheses using up to eight EMG channels, along with three neurostimulation channels.

\section{SYSTEM DESCRIPTION}

In addition to the electromechanical couplers and casing, the ALC is composed by three modules:

- Mixed Signals Processing Unit (MSPU),

- Prosthetic Control and Communication Unit (PCCU).

- Neurostimulator (NS).

These three modules were designed on dedicated Printed Circuit Boards (PCB) using commercially available integrated circuits. They comprised a stackable design with an outer round shape of $60 \mathrm{~mm}$ diameter and total height $20 \mathrm{~mm}$. Overall dimensions were chosen to fit commercially available prostheses for transhumeral amputees. The ALC system is shown in Fig. 2 with and without its case. The outer aluminum case has a $70 \mathrm{~mm}$ diameter and $30 \mathrm{~mm}$ height. Together with the OHMG and prosthetic electromechanical attachments the overall system height is $70 \mathrm{~mm}$. The core of the ALC system is the MSPU, a combination of a low-power AFE and an advanced multi-purpose MCU. The NS is managed by its own MCU, which is set as slave device of the MSPU. The PCCU directly interacts with the prosthesis but also includes other features like inertial sensors and a SD card slot.

\section{A. Mixed Signal Processing Unit (MSPU)}

The MSPU includes a low-power, low-noise AFE (ADS1299, Texas Instruments, USA) with measured Input Referred Noise of $2.58 \mu \mathrm{Vpp}$ and Common Mode Rejection Ratio of $116 \mathrm{~dB}$ [33]. It has eight independent differential channels digitized at 24-bit at different selectable sampling rates. Signals are oversampled at $1.048 \mathrm{MHz}$ and low-pass filtered with a digital third order sine decimation filter, which cut-off frequency is directly proportional to the chosen output data rate. For EMG acquisition, data rate was set on 2000 samples per second that turned in a low-pass filter at $512 \mathrm{~Hz}$. 


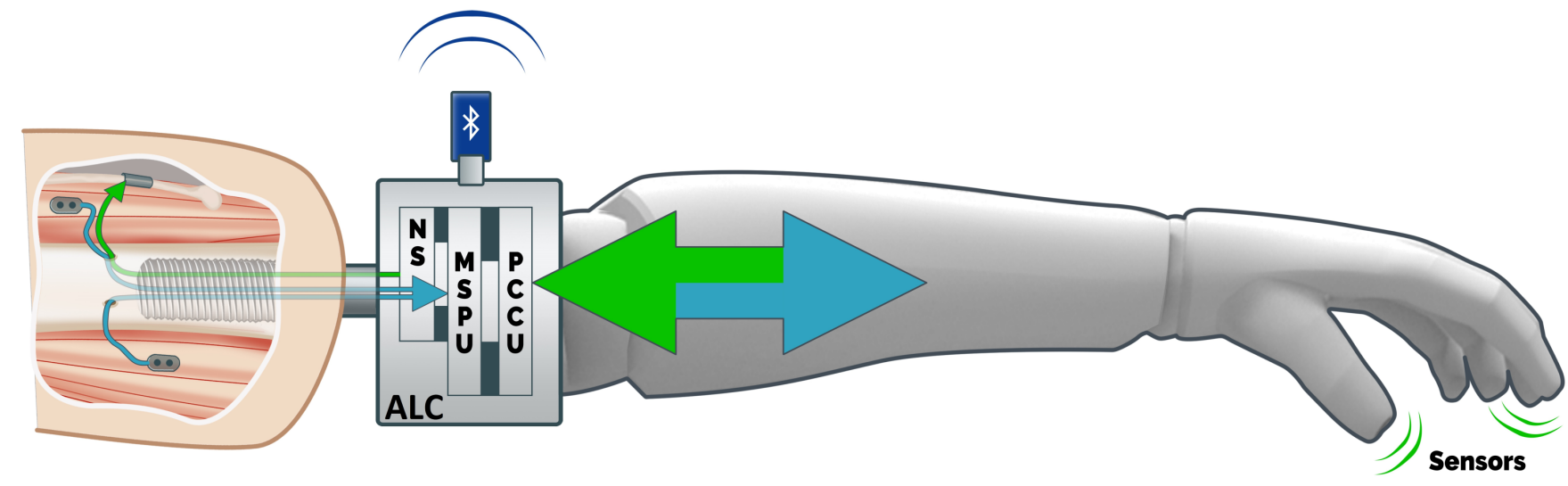

Fig. 1. Artificial Limb Controller (ALC). The system is composed by three modules: Neurostimulator (NS), Mixed Signals Processing Unit (MSPU) and Prosthetic Control and Communication Unit (PCCU). An external module can be plugged on the side of the system to achieve Bluetooth communication. Myoelectric signals are acquired from the implanted epimysial electrodes and then digitally processed to decode the motor intention of the user. In parallel, sensors on the prosthesis are periodically read and their output converted into stimulation pulses to the nerve via cuff electrode.

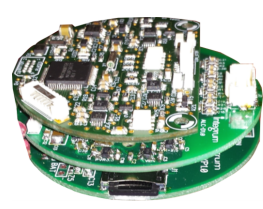

(a)

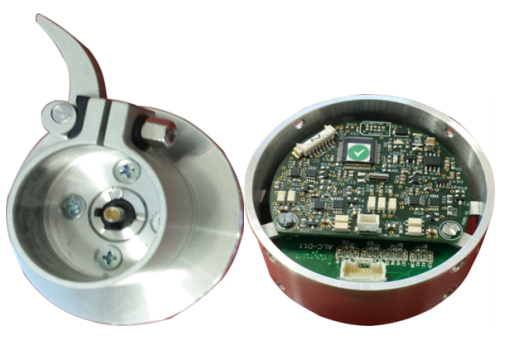

(b)

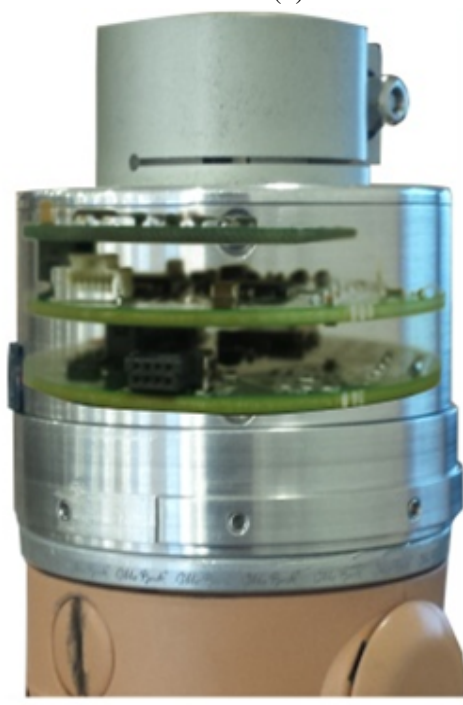

(d)
Fig. 2. Artificial Limb Controller: a) electronic modules; b) attachment device and aluminum case with electronic modules; c) Bluetooth dongle; d) representation of electronic modules inside the case.

Moreover, anti-aliasing filters $(\approx 6 \mathrm{kHz})$ were allocated in the PCB before the ADS1299 inputs. The AFE communicates with the MCU via SPI lines with a serial clock rate set at $18 \mathrm{MHz}$.

The MSPU's MCU manages the data flow between all modules. It administrates the bioelectric signal acquisition from the AFE and executes signal pre-processing (i.e., digital filters). It decodes the subject's motor intention, from building the overlapped time windows and extracting the features, to the execution of the pattern recognition algorithms. It also manages the communication and data transfer with external devices, allowing real-time visualization of bioelectric activity, as well as adjustments of control parameters from a PC or mobile device.

Considering the main characteristics of the ALC, such as wearable and capable of advanced processing, the MCU was selected giving the following features ordered by priority:

- computation capabilities (architecture and memory),

- power consumption,

- digital signal processing and compatibility with floatingpoint computations,

- peripherals and interfacing features, and

- footprint area.

There is a rather broad range of possible commercial choices in the market regarding multi-purpose $\mathrm{MCU}$, where recent ARM 32-bit cores play a leading role. The energy consumption of 8- and 16-bit cores is normally lower than 32-bit MCUs, obviously contrasting with their superior computation performance. Given their availability and superior computational power, a 32-bit core was deemed as preferable for this project if low-power consumption provided. The main MCU was chosen to be the TM4C123GH6PM (Texas Instruments, USA), based on a 32-bit ARM Cortex-M4F core. This core is the most power efficient of the Cortex-M family with a sustained processing power up to $1.25 \mathrm{DMIPS} / \mathrm{MHz}$. It has a reduced instruction set computer core based on a three-stage pipeline with separate fetch, decode, and execute units that allow most of the instructions, including multiply, to run in a single clock cycle. It also contains an embedded floating-point unit (singleprecision), an advanced high-performance bus, $256 \mathrm{~KB}$ of flash memory, and $32 \mathrm{~KB}$ of SRAM structured in Harvard architecture.

\section{B. Prosthetic Control and Communication Unit (PCCU)}

The PCCU represents the tool box of the system. The location of PCCU was strategically decided as the module closest to the prosthetic device to minimize wiring. The ALC can 
control prosthetic devices using digital and analog signals. In the first case, prosthetic movements are commanded via digital communication protocol (UART, CAN, SPI), whereas in the latter case, motors are driven by an analogue voltage signal, as commonly provided by commercially available electrodes for myoelectric prostheses. The PCCU contains the following components:

- micro SD memory card for storing usage data;

- three axis accelerometer and gyroscope;

- connector for external Bluetooth dongle;

- six analog control outputs with a range of 3.3 volts. These are buffered RC filters converting PWM signals into DC voltages;

- access to the MSPU's UART/SPI lines for digital control;

- CAN transceiver for digital control;

- audio signalling piezoelectric buzzer.

\section{Neurostimulator (NS)}

The NS module is at the top of the ALC stack in its own half-moon shaped PCB. It is connected to the MSPU via a 10-pin connector for power and communication (SPI). It utilizes an ultra-low power MCU (MSP430F2617, Texas Instruments, USA) programmed to execute and acknowledge commands sent from the MSPU. The NS ultra-low power MCU works on $500 \mu \mathrm{A}$ average current consumption, reduced by a thousand times during stand-by mode. The stimulation pulses start as voltage signals from dedicated digital-to-analog converters, and then fed into opto-isolated voltage-to-current converters which finally deliver the current stimulation pulses. In this first version of the ALC, three stimulation channels are available with independent control of pulse amplitude, width, and frequency. Each channel can output asymmetric, chargebalanced, bipolar pulses from $-500 \mu \mathrm{A}$ to $500 \mu \mathrm{A}$, limited by a $10 \mathrm{~V}$ to $-10 \mathrm{~V}$ output, and with less than $100 \mathrm{nA}$ leakage current. A blocking capacitor was placed in series with the cathode to prevent steady currents under faulty conditions. A $1 \mathrm{M} \Omega$ resistor is also present in parallel to the biological impedance and, in conjunction with the blocking capacitor, helps correct charge imbalance. The electrical circuit of each channel is represented in Fig. 3. The core of the NS stimulation circuit is a photovoltaic cell used as a controlled current source which output is modulated via a light emitting diode (LED) (photocoupler TLP3914, Toshiba). This is an entirely passive system that generates the current delivered to tissue from the conversion of LED-sourced photons to photovoltaic cells electrons with a conversion efficiency of $2.5 \% \pm 0.5 \%$. The result of such configuration is an electrically floating output with two immediate advantages: 1) improved safety, and 2) improved capability of physiological signals measurement during stimulation, as electrical isolation strongly diminishes stimulation artefacts. The major drawback is the non-linearity of the conversion from LED current to photovoltaic cell current and its sensitiveness to thermal conditions; this issue can be resolved with a careful calibration of the device. Following the standard in neurostimulation, a single stimulation event is composed by three phases:
1) stimulation pulse: cathodic current pulse with defined amplitude and width that elicits the initiation of an action potential;

2) interpulse: $50 \mu \mathrm{s}$ of zero current to allow charges to flow in the tissues [34];

3) recovery pulse: anodic reversal phase that avoids the polarization of the cells by sinking back the released current during the previous stimulation phase, and thus restoring ionic concentrations in the tissues. For charge balancing purpose, this current pulse amplitude is 10 times smaller in amplitude and 10 times longer in width. This reversal phase has been chosen slow to reduce the risk of electrode corrosion [35].

\section{FIRMWARE}

The ALC works as a Finite-State-Machine (diagram on Fig. 4) triggered by interrupts, which priorities were thoroughly defined during the design phase.

\section{A. Device Initialization}

The device initialization takes place within the first second after power on. All internal MCU peripherals are prepared for use, such as system clock, ports, and timers. The external peripherals are initialized using digital communication lines. Inertial sensors are set to output new samples at a frequency of $416 \mathrm{~Hz}$. The AFE configuration registers are written in order to setup the acquisition to 2000 SPS and gain set to 1 . The SD memory card is configured to work using the SPI protocol. Consequently, general configuration data are retrieved from memory to be available in RAM after every reboot, e.g., control and neurostimulator's settings.

\section{B. EMG Signal Acquisition and Preprocessing}

EMG signal quality is crucial in myoelectric controlled prosthesis. The ADS1299 has a data ready output signal that can be used to trigger an interrupt in the MCU for reading new samples. Although most of the applications use this approach, we preferred to trigger the reading interrupt by a timer set on the selected sampling frequency of $1000 \mathrm{~Hz}$. In this way the MCU sampling frequency can be independent from the ADS1299 output data rate and its corresponding signal bandwidth. Consequently, the sampling frequency can be easily changed externally via PC or mobile device. A lowpass filter at $524 \mathrm{~Hz}$ comes embedded with the ADS1299, while $20 \mathrm{~Hz}$ high-pass and power line notch (f $=50 \mathrm{~Hz}$, $\mathrm{Q}=35$ ) filters are implemented via firmware. In this work a simplified setup was used, such as using 2nd order filters as a compromise between signal quality and computational demands. A design choice was to apply the digital filters in a by-sample approach instead of by-window. This decision was based on the consideration that the most demanding moment in the real-time execution is when a new window has been acquired. Thus, the filters processing was distributed over all samples. 


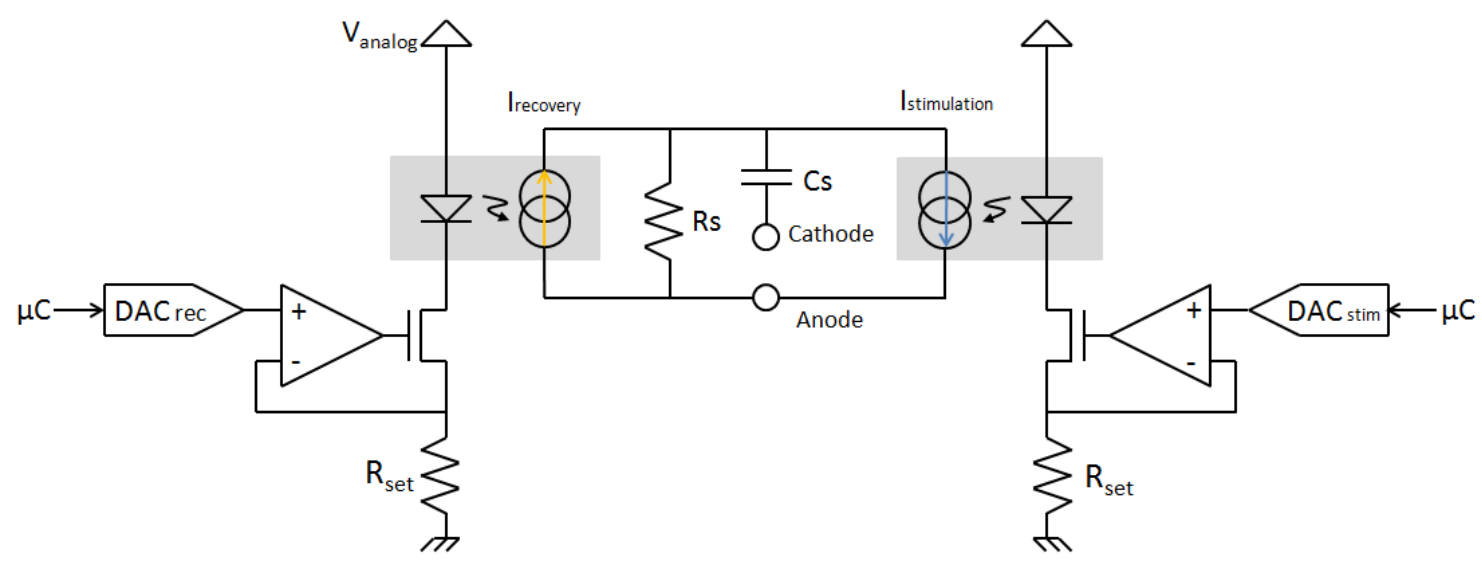

Fig. 3. Neurostimulator: one-channel electrical circuit. Two opto-isolated current sources generate a biphasic pulse. The first phase is the stimulation pulse (Istimulation, circuit on the right) and the second phase is the recovery pulse (Irecovery, circuit on the left). A blocking capacitor (Cs) is placed in series with the tissue impedance for safety purpose. The discharging resistor $(R s, 1 M \Omega)$ allows the remaining net charges on the capacitor to flow through the electrodes for charge balancing.

\section{Signal Processing, and Control Algorithms}

A standard chain of MPR data processing was implemented in the $\mathrm{MCU}$, flexible enough so that main processing parameters can be changed via external back-end. Input samples are gathered into time windows of $200 \mathrm{~ms}$ with $50 \mathrm{~ms}$ increment. Thus, a new control output is generated every $50 \mathrm{~ms}$. Common signal features are extracted from the acquired EMG, such as Mean Absolute Value (MABS), Waveform Length (WL), Slope Changes (SLPCH) and Zero Crossings (ZC) [30]. This configuration was chosen to be consistent with the literature in MPR. The extracted features are available in a feature vector ready to feed the decoding algorithm (Fig. 4). Then, the last part of the time window is slid to create overlapped windows.

Three different control algorithms were implemented and evaluated offline and in real-time, namely DC, LDA [29], and SVM [36]. DC is the standard direct control where one channel drives only one movement. It averages the signal within the time window (mean absolute value) and compares this value with a threshold for motor activation. Pattern recognition algorithms (LDA and SVM) require more processing as more features need to be extracted and the algorithmspecific computations need to be performed. They also require memory where to store coefficients needed for classification. The classifiers were trained in a PC via an open source, pattern recognition research platform named BioPatRec [37]. Successively, coefficients were downloaded into the MSPU's RAM and available for real-time classification. On-board training was not a priority at this initial stage and it is reserved for future developments. A Linear SVM was chosen for implementation, supported by the empiric consideration that it performs well enough so that a non-linear kernel transformation is unnecessary [38]. Given a feature vector $\bar{x}$, LDA and linear SVM operate the classification in a similar way (Equ. 1), their main difference is in the way they find the separating hyperplanes.

$$
f_{k}(\bar{x})=\operatorname{sign}\left(\bar{\omega}_{k} \bar{x}+b_{k}\right)
$$

where for $k$ classes: $b_{k}$ are bias terms and $\bar{\omega}_{k}$ are normal vectors to the hyperplanes. The normal vectors were precalculated in the PC in order to relieve the MCU from unnecessary recurrent calculations, as well as saving space in memory from parameters such as discriminant coefficients or support vectors. For example, the training of a SVM for the classification task addressed in our study (7 movement classes, 6 EMG channels and 4 features per EMG channel) required 12,028 bytes $\left(120\right.$ SuppVector $s^{6 \times 4}+120$ weights $\left.+7 b_{k}\right)$. By pre-calculating the normal vectors we transferred to the MCU only 679 bytes $\left(7 \bar{\omega}_{k}^{6 \times 4}+7 b_{k}\right)$, resulting in a $17: 1$ compression ratio ( $94 \%$ reduction in memory).

\section{Control and Communication Mode}

The ALC is meant to work in two modalities: control and communication (Fig. 4). The default control-mode imposes the device to cyclically execute the control task looping between acquiring a new time window, extracting the features, classification, reading sensors, updating the stimulation and executing the movement. Also, a SD card is used, triggered by a timer, to continuously log data with the aim of monitoring all relevant process. The values of the predicted movements are stored along with extracted features, hand force sensors, accelerometer, gyroscope, temperature and battery voltage. This data can be later analyzed in order to better understand prosthetic use and for the evaluation of potential sources of errors.

The system was designed to wirelessly interface a PC or mobile devices for prosthetic fitting, monitoring and data management via Bluetooth link. An external communication board (Fig. 2-c) must be connected to the main stack as represented in Fig. 1 to enable wireless communication. By doing this, the control-mode can be paused switching the device to the communication-mode. Thus, the system stays in idle state waiting to receive commands via Bluetooth and consequently execute them before returning to idle state. Several commands are available and can be utilized mostly by technicians for testing purposes. Commands can vary from reading firmware version, enabling/disabling filters, streaming 


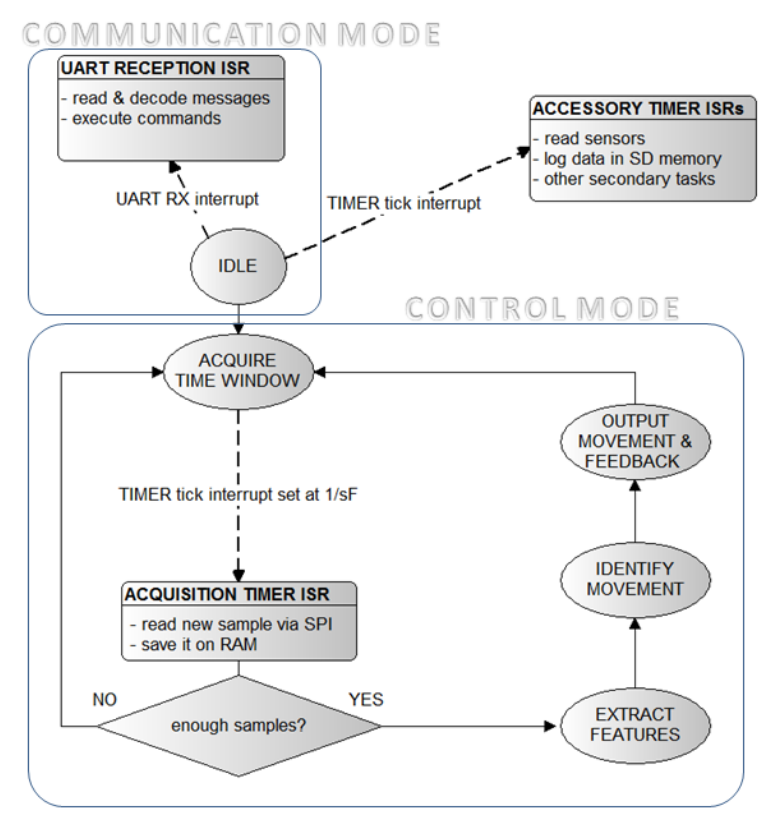

Fig. 4. Finite-State-Machine diagram. The Artificial Limb Controller can be operated in two different modes: control and communication. The default control-mode imposes the device to cyclically execute the control task looping between acquiring a new time window, extracting the features, performing the classification, reading sensors and updating the stimulation, and executing the classified movement. The control-mode can be paused switching the device to the communication-mode. Thus, it stays in idle state waiting to receive and execute commands sent by external connected devices. Communication-mode is especially used for read and modify settings or test purposes.

out EMG signals or the classifier prediction outputs, and updating control parameters like changing thresholds or load new pattern recognition coefficients.

\section{E. Neurostimulation}

While the control procedure is cyclically repeated, sensors in the robotic hand must be processed and converted into stimulation pulses to elicit the perception of touch.

The robotic devices utilized for this experiment were provided by Ottobock (Vienna, Austria): a 12K50 myoelectric elbow and a SensorHand. The SensorHand has three embedded pressure sensors that are available to be read only if the device is correctly switched into digital mode via a proprietary communication protocol.

An intuitive stimulation pattern was implemented where the amplitude and pulse-width are constant while the frequency varies proportionally with the grasping force. In this approach the higher the pressure, the higher the stimulation frequency (stronger sensation) (Fig. 5). For safety reasons, it was decided to limit the frequency of stimulation to $30 \mathrm{~Hz}$ as this system is aimed for chronic use [39].

\section{EXPERIMENTAl TeSts AND Results}

\section{A. Specification and Power Consumption}

The ALC specifications are given in Table I. The ALC draws different currents depending on the operation mode. When the Bluetooth module is plugged and data is streamed to an external device, the ALC drains $90 \mathrm{~mA}$. More importantly,

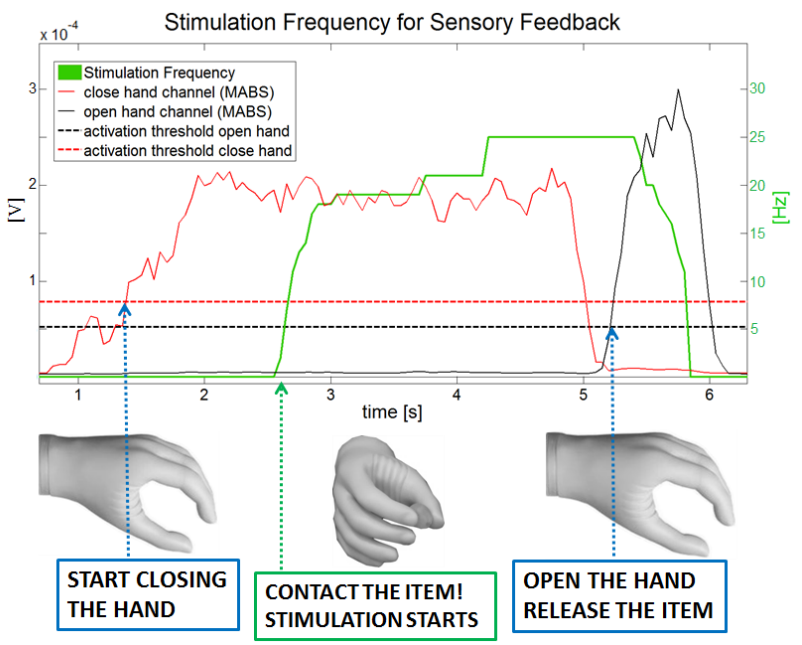

Fig. 5. Direct neural sensory feedback. The stimulation pulse amplitude and width were constant while the frequency varied proportionally to the grasping force. In the current approach at a higher pressure, a higher stimulation frequency (stronger sensation). This figure shows an example in which as soon as the muscle's contraction reached the activation threshold, the hand started to close until making contact with an item, at which point the stimulation frequency was increased proportionally to the force applied. In the last part, the user opens the prosthetic hand to release the item.

when the system works in the standard control-mode it needs a minimum current of $65 \mathrm{~mA}$, which is reduced to $50 \mathrm{~mA}$ in idle mode. The battery life for a standard prosthetic setup combined with the ALC was found to be 15 to 18 hours per day during four weeks out-of-the-lab verification period (more details about the setup are provided in section V-D).

TABLE I

SPECIFICATIONS OF THE ARTIFICIAL Limb CONTROLLER

\begin{tabular}{|c|c|c|c|}
\hline \multicolumn{4}{|c|}{ General Input Specifications } \\
\hline Differential Channels & Sampling Resolution [bit] & IRN $[\mu \mathrm{Vpp}]$ & CMRR $[\mathrm{dB}]$ \\
\hline 8 & 24 & 2.58 & 116 \\
\hline \multicolumn{4}{|c|}{ Stimulation } \\
\hline Channels & \multicolumn{3}{|c|}{ Settable Parameters } \\
\hline 3 & $\begin{array}{c}\text { Current }[\mu \mathrm{A}] \\
10 \text { to } 500 \\
\text { with steps of } 10\end{array}$ & $\begin{array}{c}\text { Pulse-Width }[\mu \mathrm{s}] \\
50 \text { to } 500 \\
\text { with steps of } 10\end{array}$ & $\begin{array}{c}\text { Frequency }[\mathrm{Hz}] \\
1 \text { to } 100\end{array}$ \\
\hline \multicolumn{4}{|c|}{ Control } \\
\hline \multicolumn{4}{|c|}{$6 \times$ Analog Outputs, CAN, SPI, $2 \times$ UART } \\
\hline \multicolumn{4}{|c|}{ Others } \\
\hline \multicolumn{4}{|c|}{ SD card, 3 axis Gyroscope + Accelerometer, piezoelectric buzzer, Bluetooth capability } \\
\hline \multicolumn{4}{|c|}{ Power Consumption } \\
\hline Supply Voltage [V] & Idle Mode $[\mathrm{mA}]$ | Control & Mode $[\mathrm{mA}] \mid$ Stre & reaming Data (Bluetooth) $[\mathrm{mA}]$ \\
\hline$\geq 5.5$ & 50 & 65 & 90 \\
\hline
\end{tabular}

\section{B. Microcontroller Tasks Analysis}

Time characterization of all processing routines is reported in Table II. The most frequent deadline is the interrupt of the sampling timer, and the most computations are required when a new time window is ready to be processed (every 50 ms). As expected, perfect schedulability was hard to achieve. The ALC firmware in this particular implementation was not able to perform all control related tasks within the time before a new sample is ready to be read in the AFE (1 ms). 
The time required by features extraction, classification and execution grows proportionally with the number of channels used. However, with the maximum number of channels (eight), this time was found to be around three milliseconds, which means that only three samples every new time window are discarded in order to complete the control task.

TABLE II

MCU TASK TIMING FOR 8 CHANNELS AND 7 MOVEMENTS

\begin{tabular}{|c|c|c|c|c|}
\hline & Task & \multicolumn{3}{|c|}{$\begin{array}{c}\text { Execution time } \\
{[\mu \mathrm{s}]}\end{array}$} \\
\hline \multirow{5}{*}{ 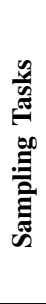 } & $\begin{array}{l}\text { Read data } \\
\text { from SPI }\end{array}$ & \multicolumn{3}{|c|}{22.5} \\
\hline & \multirow[t]{2}{*}{ Filter data } & $\begin{array}{c}\text { Notch } \\
11\end{array}$ & & $\begin{array}{l}\text { igh-Pass } \\
10.5\end{array}$ \\
\hline & & \multicolumn{3}{|c|}{21.5} \\
\hline & Store in RAM & \multicolumn{3}{|c|}{2.4} \\
\hline & SCHEDULABILITY & \multicolumn{3}{|c|}{$46.4 / 10^{3}$} \\
\hline \multirow{7}{*}{$\begin{array}{l}\frac{\tilde{y}}{\tilde{y}} \\
\frac{\tilde{\theta}}{0} \\
\frac{\dot{E}}{0} \\
0\end{array}$} & Slide window & \multicolumn{3}{|c|}{316} \\
\hline & \multirow{3}{*}{ Extract features } & $\begin{array}{c}\text { MABS } \\
286\end{array}$ & & $\begin{array}{l}\text { WL } \\
560\end{array}$ \\
\hline & & $\begin{array}{l}\mathrm{ZC} \\
992\end{array}$ & & $\begin{array}{l}\text { SPCH } \\
1150\end{array}$ \\
\hline & & \multicolumn{3}{|c|}{2990} \\
\hline & Classify movement & $\begin{array}{l}\text { LDA } \\
25\end{array}$ & & $\begin{array}{c}\text { SVM } \\
43\end{array}$ \\
\hline & Execute movement & \multicolumn{3}{|c|}{2.7} \\
\hline & SCHEDULABILITY & \multicolumn{3}{|c|}{$3 \cdot 10^{3} / 50 \cdot 10^{3}$} \\
\hline \multirow{3}{*}{ 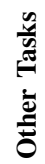 } & \multirow{2}{*}{ Read from Sensors } & $\begin{array}{l}\text { IMU } \\
47.4\end{array}$ & $\begin{array}{l}\text { Hand } \\
5\end{array}$ & $\begin{array}{c}\text { System } \\
20\end{array}$ \\
\hline & & \multicolumn{3}{|c|}{52.4} \\
\hline & Store in SD Memory & \multicolumn{3}{|c|}{188} \\
\hline
\end{tabular}

\section{Myoelectric Pattern Recognition Performance}

The evaluation of EMG feature extraction was conducted in the ALC and in a PC running BioPatRec using a pre-recorded data set. No relevant discrepancy between embedded and PC arithmetic was found (absolute error around $10^{-10}$ ).

The MPR algorithms were tested offline and in real-time by eight able-bodied subjects, a congenital transradial amputee, and the OHMG pilot patient. Subjects were in a range of $29.2 \pm 6.1$ years old. The able-bodied and transradial subjects were prepared with six pairs of surface EMG electrodes $(\mathrm{Ag} / \mathrm{AgCl})$ for differential recordings equally spaced around the most proximal third of the forearm. In the case of the OHMG pilot patient, the ALC was directly connected to the OHMG implant, comprising of two bipolar and three monopolar epimysial electrodes for control, and one cuff electrode with three sites for stimulation. The target movements were: hand open-close, hand flex-extend, pro/supination of the wrist, and no movement. For the transhumeral OHMG patient, hand flex-extend was replaced with elbow flex-extend.

For all subjects, the Bluetooth dongle was plugged in the side of the ALC's case to achieve a wireless link with a PC. All

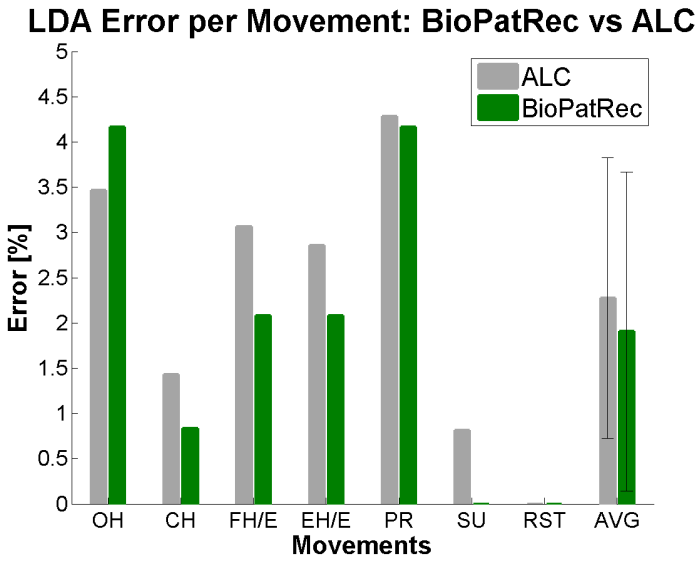

(a)

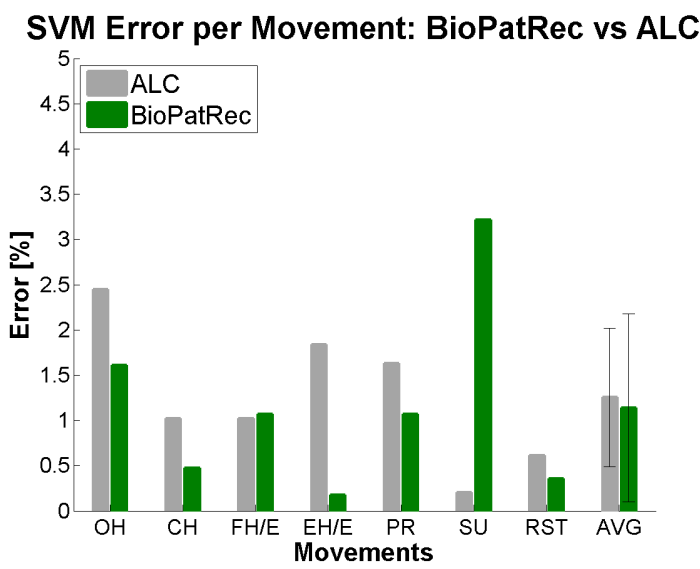

(b)

Fig. 6. Comparison of offline tests between the ALC and BioPatRec. The figure shows error percentages for Linear Discriminant Analysis (a) and Support Vector Machine (b) for all subjects and all movements $(\mathrm{OH}=$ open hand, $\mathrm{CH}=$ close hand, $\mathrm{FH} / \mathrm{E}=$ flex hand or elbow, $\mathrm{EH} / \mathrm{E}=$ extend hand or elbow, $\mathrm{PR}=$ pronation of the hand, $\mathrm{SU}=$ supination of the hand, RST $=$ rest movement, $\mathrm{AVG}=$ average $)$. In the case of the OHMG pilot patient flexion and extension of the hand were replaced with elbow movements. No statistically significant difference between averages was found $(p=0.77)$.

recordings were taken with BioPatRec software and streaming the EMG data out of the ALC. EMG activity was visually inspected in all channels before proceeding to the recording phase. Then, subjects were sat in a comfortable position in front of the PC and asked to follow instructions from the BioPatRec software. Each movement was executed three times per recording, alternating three seconds of contraction with three seconds of relaxation. Training coefficients and offline accuracies were processed on BioPatRec using $40 \%$ of data for training, $20 \%$ for validation and $40 \%$ for test. The same test sets were also used to process the offline accuracies on the ALC. Results are compared in Fig. 6. In average, accuracies for both algorithms were found to be around $98 \%$. Also, no statistically significant difference (Wilcoxon Signed Rank test, $p=0.77$ ) was found between averages accuracies on the ALC and PC, confirming high performances of the floating-point computations on the MCU.

Offline accuracy was also compared acquiring the signal at different frequencies. Test was run at $500 \mathrm{~Hz}, 1 \mathrm{kHz}$ and 
$2 \mathrm{kHz}$, and following the same recording protocol aforementioned. Average offline accuracies are presented in Table III; no statistically significant difference was found between the averages (Friedmans's test, $p=0.14$ ). Results are inline with previous studies by others [25], [40].

The main focus of our validation procedure was the realtime performance of the system. BioPatRec interface was adapted to read the prediction outputs from the ALC. The Motion Test was chosen as the most appropriate test to show realtime classification performance [8]. In this test, subjects were asked to perform movements randomly prompted on a screen. For every movement requested to perform, 20 noncontiguous cycles of the classifier prediction had to be correct within 10 seconds (200 classifier cycles) to consider the particular movement completed [41]. Each one of the implemented algorithms was tested in separate Motion Tests. The order of execution was randomized between participants to avoid any significant impact related to familiarization with the test. Results are reported in Fig. 7. LDA and SVM had comparable performance with average completion rates above 98\%. In average, around nine misclassifications happened before the first correct prediction. Moreover, completion time shows that generally 35 predictions were executed before completing the task.

TABLE III

Average OfFline ACCURACy Related to SAmpling Frequency

\begin{tabular}{c|c|c}
\hline \hline \multirow{2}{*}{ Sampling Frequency [Hz] } & \multicolumn{2}{|c}{$\begin{array}{c}\text { Accuracy } \\
{[\%]}\end{array}$} \\
\cline { 2 - 3 } & LDA & SVM \\
\hline 2000 & 100 & $99.1(\operatorname{std} 0.2)$ \\
\hline 1000 & 100 & $99.9(\operatorname{std} 0.2)$ \\
\hline 500 & $98.8(\operatorname{std} 2.1)$ & $98.2(\operatorname{std} 3.9)$ \\
\hline \hline
\end{tabular}

\section{Neurostimulation and out-of-the-lab Verification}

To validate the closed-loop control the OHMG pilot patient was asked to grasp delicate objects while blindfolded and wearing isolating headphones. The ALC was configured in proportional DC and the patient was left to try the sensory stimulation while grabbing different objects for approximately five minutes prior evaluation. Selected items were eggs and grapes. The patient managed to gently grasp each object eight times, in two testing sessions, without breaking or damaging them (32 success grasps out of 32 attempts).

The OHMG pilot patient was provided with the ALC for four weeks for regular use in his daily activities. The neurostimulator was not included in the system for this trial. The pilot patient was already using a myoelectric prosthesis with an analog controller which provided the same functions as commercially available myoelectric prosthetic electrodes. A simpler but more robust robotic hand was preferred (VaryPlus, OttoBock, Germany), sacrificing multiple gestures in favor of a stronger and reliable power grasp (one degree of freedom). Wrist rotation was avoided for the moment but considered for future upgrades. The importance of a myoelectric elbow lock/unlock switch in a transhumeral amputation was highly emphasized by the OHMG pilot patient and thus implemented for daily use. Finally, the ALC was set with proportional DC over triceps and biceps for open and close hand respectively, combined with elbow lock/unlock by co-contraction. The subject used the ALC for four weeks while real-time data was continuously stored in the SD card for post-analysis.

\section{DISCUSSION}

Offline and real-time validation of classification algorithms played a crucial role in the analysis of the ALC. Despite the single-precision arithmetic, the ALC was able to classify seven different movements with an accuracy comparable to that of a PC. Moreover, the Motion Tests showed high rate of correct predictions for both classifiers. The ALC was able to functionally provide sensory information thanks to the neurostimulator module. The system was also validated with the OHMG pilot patient during a preliminary four weeks outof-the-lab test. Here, battery life was found to be 15 to 18 hours using a standard prosthetic device battery with capacity of $800 \mathrm{mAh}$. It is worth to note that the neurostimulator was excluded from this particular test and that the control was based simply on two input channels (DC). The ALC power consumption was anyway consistent with bench tests (Table I). Also, no power optimization was implemented in the current firmware, which will be a subject for future improvements.

The task timing test (Table II) shows the real-time behavior of the ALC. As expected from the choice of using floatingpoint arithmetic, perfect schedulability of all tasks was not achieved. In fact, features extraction and movement classification cannot be executed within the time that a new sample is ready from the ADS1299. Processing delay resulted anyway consistent with what reported in similar studies. Around three milliseconds need to be invested for completing all control related tasks. A limitation of the current implementation is that EMG acquisition is temporary paused within that processing delay. An optimization can be done on this side, such as collecting also those samples that arrive while the control related tasks are still under execution. Integer arithmetic could be also implemented to establish a complete performance comparison with floating-point computation.

Purposely, no post-processing algorithms were applied to mitigate misclassifications as our intent was to show the classification capability of the algorithms only. Nevertheless, current feature extraction and classification time represent a small enough delay as to allow further algorithms to produce a smooth control output, such as majority vote or velocity ramp [42].

The offline accuracy for both algorithms did not change consistently when the sampling frequency was reduced to 500 $\mathrm{Hz}$. Even though this must be confirmed with real-time test, it could mean that the acquisition rate can be slowed down providing a wider time frame for combining the classification with further advanced signal processing.

Digital filters implementation can be further improved. Table II shows the abundant free processing time between each sample. This time could be spent for more advanced 


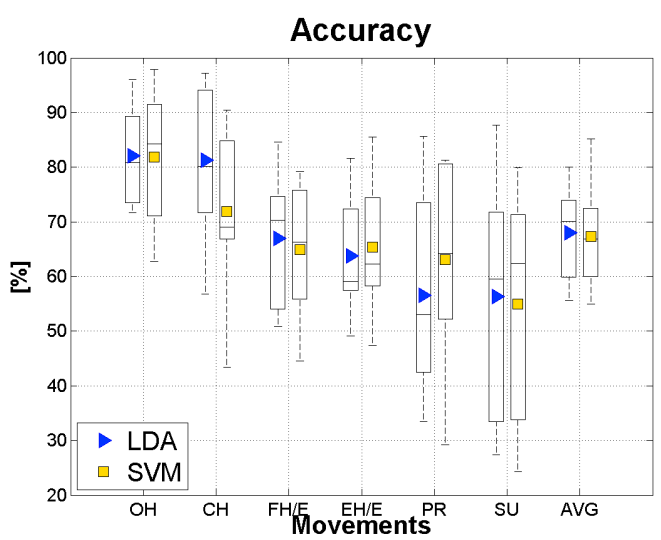

(a)

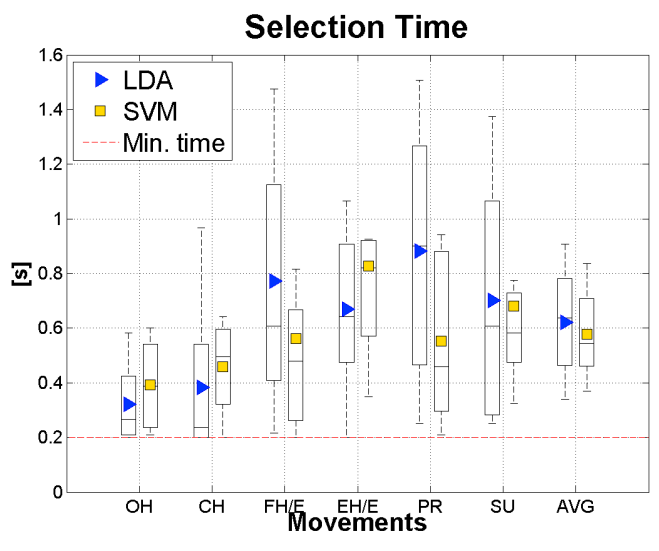

(c)

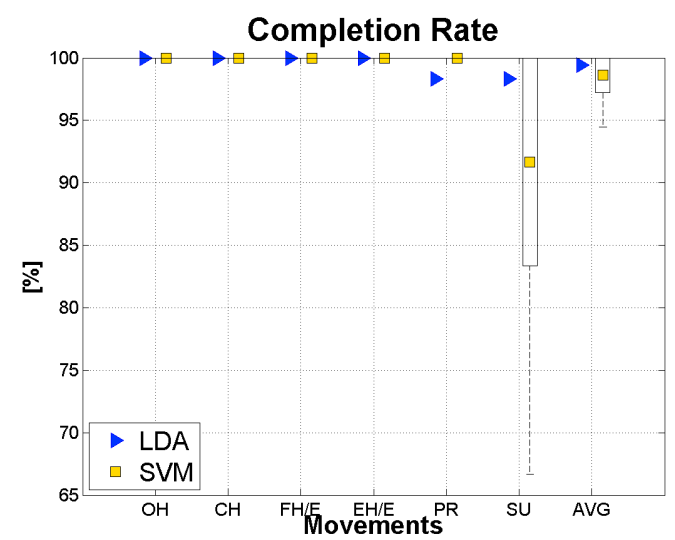

(b)

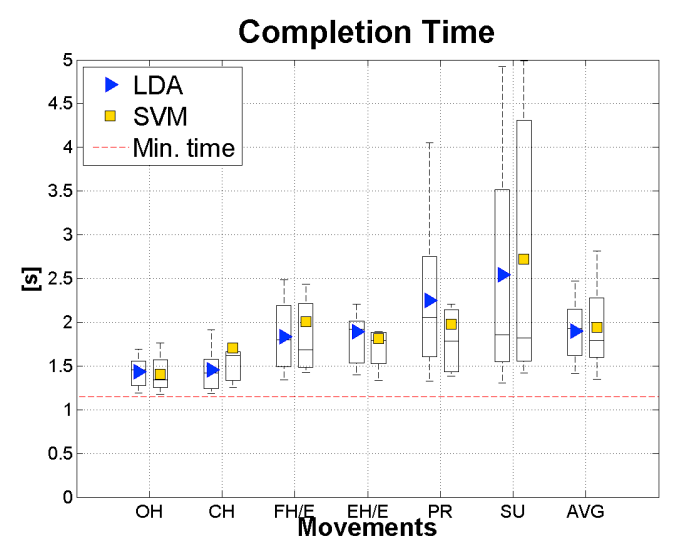

(d)

Fig. 7. Real-time test of the ALC classifications. Motion Test results, in a boxes and whiskers format, for all subjects and all movements. The symbol and the line represent respectively the mean and median of each box. Accuracy (a), was calculated using the predictions during the completion time, and only completed motions contributed. Completion Rate (b) is the rate of successful trials. Selection Time (c) is the time required to reach the first correct prediction (minimum time is equal to the processing time window). Completion Time (d) is the time to reach 20 correct predictions (minimum time is given by the sum of a full time window and 19 incremental windows). Note that all minimum times shown are calculated not taking into account the time needed for processing and wireless transmission of the classification result.

processing or, if battery life becomes priority, the ALC can be set in sleep mode to save energy. Similarly, higher order by-window FIR filters will be tested in the future.

Inertial sensors were included in the system to complement information on prosthetic use, and to potentially improve the controllability of the system by incorporating such information into the motor volition decoding. At this point this information was not used in the control strategies but is available for future developments.

Prosthetic control via electroneurography (ENG) signals represents an interesting opportunity enabled by the OHMGALC system, and will be explored in future work. The ALCs analog front-end allows for ENG recordings and whether it can reliably extract extraneural activity in activities of the daily living using a cuff electrodes is under investigation. In such case different signal pre-processing, features extraction, and control algorithms will have to be implemented and validated in this platform.

\section{CONCLUSION}

An embedded digital controller was implemented in this work aiming to exploit the advantages of the OHMG technology. This system allows for bioelectric signals acquisition, processing, decoding of motor intent, prosthetic control and sensory feedback. Standard myoelectric control (direct control) was implemented as well as two robust pattern recognition algorithms. It includes a neurostimulator to provide direct neural feedback aimed for restoration of tactile sensations. Hardware was thoroughly bench tested and validated in terms of real-time tasks characterization and power consumption. Pattern recognition accuracy was tested, both offline and realtime, showing promising results for clinical implementation. Functionality was finally proven in a first pilot patient allowing him to blindly grasp delicate objects. The system was designed to be reliably used in activities of daily living, as well as a research platform to monitor prosthesis usage and training, machine learning based control techniques, and neural stimulation paradigms. 


\section{ACKNOWLEDGMENT}

The authors thank the Swedish Research Council (Vetenskapsrdet), VINNOVA, the EU project DeTOP, all volunteers who have taken part in the recording sessions, Jason Millenaar for designing system's enclosure and illustration, Alejandra Zepeda, Fredrik Ekasen, Jurek Lamkiewicz, and Janos Kalmar for their valuable help during this work.

\section{REFERENCES}

[1] M. Johannes, J. Bigelow, J. Burck, S. Harshbarger, M. Kozlowski, and T. Van Doren, "An overview of the developmental process for the modular prosthetic limb," Johns Hopkins APL Technical Digest (Applied Physics Laboratory), vol. 30, no. 3, pp. 207-216, 2011.

[2] R. Wirta, D. Taylor, and F. Finley, "Pattern-Recognition Arm Prosthesis: a Historical Porspective - a final Report," Bulletin of Prosthetic Research Journal, vol. 10, no. 30, pp. 8-35, 1978.

[3] K. Østlie, I. Lesjø, R. Franklin, B. Garfelt, O. Skjeldal, and P. Magnus, "Prosthesis rejection in acquired major upper-limb amputees: a population-based survey," Disability and Rehabilitation: Assistive Technology, vol. 7, no. 4, pp. 294-303, 2012.

[4] L. Pezzin, T. Dillingham, E. MacKenzie, P. Ephraim, and P. Rossbach, "Use and satisfaction with prosthetic limb devices and related services," Archives of Physical Medicine and Rehabilitation, vol. 85, no. 5, pp. 723-729, 2004.

[5] D. Farina, N. Jiang, H. Rehbaum, A. Holobar, B. Graimann, H. Dielt, and O. Aszmann, "The Extraction of Neural Information from the Surface EMG for the Control of Upper-Limb Prostheses: Emerging Avenues and Challenges," IEEE Transactions on Neural Systems and Rehabilitation Engineering, vol. 22, no. 4, pp. 797-809, 2014.

[6] A. Andrade, A. Pereira, S. Walter, R. Almeida, R. Loureiro, D. Compagna, and P. J. Kyberd, "Bridging the gap between robotic technology and health care," Biomedical Signal Processing and Control, vol. 10, no. 1, pp. 65-78, 2014.

[7] O. Aszmann, A. Roche, S. Salminger, T. Paternostro-Sluga, M. Herceg, A. Sturma, C. Hofer, and D. Farina, "Bionic reconstruction to restore hand function after brachial plexus injury: A case series of three patients," The Lancet, vol. 385, no. 9983, pp. 2183-2189, 2015.

[8] T. Kuiken, G. Li, B. Lock, R. Lipschutz, L. Miller, K. Stubblefield, and K. Englehart, "Targeted muscle reinnervation for real-time myoelectric control of multifunction artificial arms," Journal of the American Medical Association, vol. 301, no. 6, pp. 619-628, 2009.

[9] T. Kung, R. Bueno, G. Alkhalefah, N. Langhals, M. Urbanchek, and P. Cederna, "Innovations in prosthetic interfaces for the upper extremity." Plastic and Reconstructive Surgery, vol. 132, no. 6, pp. 1515-1523, 2013.

[10] K. Hagberg, E. Häggström, M. Uden, and R. Brånemark, "Socket versus bone-anchored trans-femoral prostheses: Hip range of motion and sitting comfort," Prosthetics and Orthotics International, vol. 29, no. 2, pp. 153-163, 2005.

[11] R. Brånemark, Ö. Berlin, K. Hagberg, P. Bergh, B. Gunterberg, and B. Rydevik, "A novel osseointegrated, percutaneous prosthetic system for treatment of patients with transfemoral amputation: A prospective study of 51 patients," The Bone and Joint Journal, vol. 96-B, no. 1, pp. 106-113, 2014.

[12] M. Ortiz-Catalan, B. Håkansson, and R. Brånemark, "An osseointegrated human-machine gateway for long-term sensory feedback and motor control of artificial limbs," Science Translational Medicine, vol. 6, no. 257, p. 257re6, 2014.

[13] X. Navarro, T. Krueger, N. Lago, S. Micera, T. Stieglitz, and P. Dario, "A critical review of interfaces with the peripheral nervous system for the control of neuroprostheses and hybrid bionic systems," Journal of the Peripheral Nervous System, vol. 10, no. 3, pp. 229-258, 2005.

[14] M. Ortiz-Catalan, R. Brånemark, B. Håkansson, and J. Delbeke, "On the viability of implantable electrodes for the natural control of artificial limbs: Review and discussion," BioMedical Engineering OnLine, vol. 11, no. 1, pp. 33-57, 2012.

[15] F. Clippinger, R. Avery, and B. Titus, "A sensory feedback system for an upper-limb amputation prosthesis," Bulletin of Prosthetics Research, vol. 10, no. 22, pp. 247-258, 1974.

[16] J. Wright, V. Macefield, A. Schaik, and J. Tapson, "A Review of Control Strategies in Closed-Loop Neuroprosthetic Systems," Frontiers in Neuroscience, vol. 10, no. 312, pp. 1-13, 2016.
[17] D. W. Tan, M. A. Schiefer, M. W. Keith, J. R. Anderson, J. Tyler, and D. J. Tyler, "A neural interface provides long-term stable natural touch perception," Science Translational Medicine, vol. 6, no. 257, p. $257 \mathrm{ra} 138$.

[18] C. M. Oddo, S. Raspopovic, F. Artoni, A. Mazzoni, G. Spigler, F. Petrini, F. Giambattistelli, F. Vecchio, F. Miraglia, L. Zollo, G. Di Pino, D. Camboni, M. C. Carrozza, E. Guglielmelli, P. M. Rossini, U. Faraguna, and S. Micera, "Intraneural stimulation elicits discrimination of textural features by artificial fingertip in intact and amputee humans," eLife, vol. 5, 2016.

[19] T. S. Davis, H. A. C. Wark, D. T. Hutchinson, D. J. Warren, K. O’Neill, T. Scheinblum, G. A. Clark, R. A. Normann, and B. Greger, "Restoring motor control and sensory feedback in people with upper extremity amputations using arrays of 96 microelectrodes implanted in the median and ulnar nerves," Journal of Neural Engineering, vol. 13, no. 3, p. 036001 .

[20] P. Herberts, C. Almström, R. Kadefors, and P. Lawrence, "Hand prosthesis control via myoelectric patterns." Acta Orthopaedica Scandinavica Journal, vol. 44, no. 4, pp. 389-409, 1973.

[21] E. Scheme and K. Englehart, "Electromyogram pattern recognition for control of powered upper-limb prostheses: State of the art and challenges for clinical use," Journal of Rehabilitation Research and Development, vol. 48 , no. 6, pp. 643-659, 2011.

[22] F. Tenore, R. Armiger, R. Vogelstein, D. Wenstrand, S. Harshbarger, and K. Englehart, "An embedded controller for a 7-degree of freedom prosthetic arm," Proceedings for the IEEE Engineering in Medicine and Biology Society Conference, pp. 185-188, 2008.

[23] T. Hirata, T. Nakamura, R. Kato, S. Morishita, and H. Yokoi, "Development of mobile controller for EMG prosthetic hand with tactile feedback," Proceedings for the IEEE/ASME International Conference on Advanced Intelligent Mechatronics, pp. 110-115, 2011.

[24] H. Liu, D. Yang, L. Jiang, and S. Fan, "Development of a multi-DOF prosthetic hand with intrinsic actuation, intuitive control and sensory feedback," Industrial Robot-an International Journal, vol. 41, no. 4, pp. 381-392, 2014.

[25] S. Benatti, F. Casamassima, B. Milosevic, E. Farella, P. Schonle, S. Fateh, T. Burger, Q. Huang, and L. Benini, "A Versatile Embedded Platform for EMG Acquisition and Gesture Recognition," IEEE Transactions on Biomedical Circuits and Systems, vol. 9, no. 5, pp. 620-630, 2015.

[26] Z. Xiaorong, H. He, and Q. Yang, "Implementing an FPGA system for real-time intent recognition for prosthetic legs," Proceedings for the Design Automation Conference, pp. 169-175, 2012.

[27] A. Boschmann, A. Agne, L. Witschen, G. Thombansen, F. Kraus, and M. Platzner, "FPGA-based Acceleration of High Density Myoelectric Signal Processing," Proceedings for the ReConFigurable Computing and FPGAs Conference, 2015.

[28] R. Merletti, A. Botter, A. Troiano, E. Merlo, and M. Minetto, "Technology and instrumentation for detection and conditioning of the surface electromyographic signal: state of the art," Clinical Biomechanics, vol. 24, no. 2, pp. 122-134, 2009.

[29] K. Englehart and B. Hudgins, "A robust, real-time control scheme for multifunction myoelectric control," IEEE Transactions on Biomedical Engineering, vol. 50, no. 7, pp. 848-854, 2003.

[30] B. Hudgins, P. Parker, and R. Scott, "A new strategy for multifunction myoelectric control," IEEE Transactions on Biomedical Engineering, vol. 40, no. 1, pp. 82-94, 1993.

[31] A. Fougner, E. Scheme, A. Chan, K. Englehart, and Ø. Stavdahl, "Resolving the limb position effect in myoelectric pattern recognition," IEEE Transactions on Neural Systems and Rehabilitation Engineering, vol. 19, no. 6, pp. 644-651, 2011.

[32] G. Rasool, K. Iqbal, N. Bouaynaya, and G. White, "Real-Time Task Discrimination for Myoelectric Control Employing Task-Specific Muscle Synergies," IEEE Transactions on Neural Systems and Rehabilitation Engineering, vol. 24, no. 1, pp. 98-108, 2016.

[33] E. Mastinu, M. Ortiz-catalan, and B. Håkansson, "Analog Front-Ends comparison in the way of a portable, low-power and low-cost EMG controller based on Pattern Recognition," Proceedings for the IEEE Engineering in Medicine and Biology Society Conference, pp. 2111$2114,2015$.

[34] P. Gorman and J. Mortimer, "The Effect of Stimulus Parameters on the Recruitment Characteristics of Direct Nerve Stimulation," IEEE Transactions on Biomedical Engineering, vol. BME-30, no. 7, pp. 407414, 1983.

[35] D. Merrill, M. Bikson, and J. Jefferys, "Electrical stimulation of excitable tissue: Design of efficacious and safe protocols," Journal of Neuroscience Methods, vol. 141, no. 2, pp. 171-198, 2005. 
[36] M. Oskoei and H. Hu, "Support Vector Machine-Based Classification Scheme for Myoelectric Control Applied to Upper Limb," IEEE Transactions on Biomedical Engineering, vol. 55, no. 8, pp. 1956-1965, 2008.

[37] M. Ortiz-Catalan, R. Brånemark, and B. Håkansson, "BioPatRec: A modular research platform for the control of artificial limbs based on pattern recognition algorithms," Source Code for Biology and Medicine, vol. 8, pp. 11-29, 2013.

[38] Z. Khokhar, Z. Xiao, and C. Menon, "Surface EMG pattern recognition for real-time control of a wrist exoskeleton," Biomedical Engineering online, vol. 9, no. 1, p. 41, 2010.

[39] D. McCreery, W. Agnew, T. Yuen, and L. Bullara, "Relationship between stimulus amplitude, stimulus frequency and neural damage during electrical stimulation of sciatic nerve of cat," Medical and Biological Engineering and Computing, vol. 33, no. 3, pp. 426-429, 1995.

[40] G. Li, Y. Li, L. Yu, and Y. Geng, "Conditioning and sampling issues of EMG signals in motion recognition of multifunctional myoelectric prostheses," Annals of Biomedical Engineering, vol. 39, no. 6, pp. 17791787, 2011.

[41] M. Ortiz-Catalan, B. Håkansson, and R. Brånemark, "Real-time and simultaneous control of artificial limbs based on pattern recognition algorithms," IEEE Transactions on Neural Systems and Rehabilitation Engineering, vol. 22, no. 4, pp. 756-764, 2014.

[42] A. Simon, L. Hargrove, B. Lock, and T. Kuiken, "A decision-based velocity ramp for minimizing the effect of misclassifications during realtime pattern recognition control," IEEE Transactions on Biomededical Engineering, vol. 29, no. 8, pp. 997-1003, 2011.

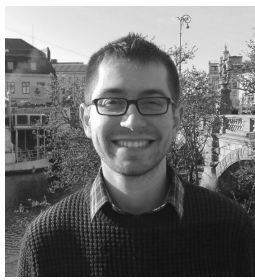

Enzo Mastinu (S'15) received his M.Sc. degree with top grade in Electronic Engineering from Universitá degli Studi di Cagliari, Italy, in 2014. His M.Sc. thesis was awarded at the "Innovation Day Design Contest 2014", Milan, Italy. During and after the M.Sc. he worked as HW/FW designer for a spin-off company and a postgraduate research scholarship, respectively.

$\mathrm{He}$ is currently an industrial Ph.D. student at the Department of Electrical Engineering of Chalmers University of Technology and Integrum AB, Gothenburg, Sweden. His research focuses on embedded systems for prosthetic control, mainly regarding upper limb amputees. Research interests cover bioelectric signal acquisition and processing, electronics design, algorithms for pattern recognition, as well as bone-anchored prostheses and osseointegration.

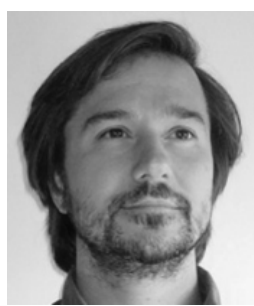

Pascal Doguet received his M.Sc. degree and $\mathrm{Ph} . \mathrm{D}$. in Electronic Engineering from Université Catholique de Louvain, Belgium, in 2000. He also completed a European M.Sc. degree in Renewable Energy in 2004. He devoted most of his 20 years career in biomedical engineering and, especially, neurostimulation. He designed and developed several active implantable medical devices, such as a prosthesis for the blind and neurostimulators for sleep apnea, deep brain stimulation and epilepsy.

Pascal Doguet is the founder and Chief Technology Officer of Synergia Medical [www.synergiam.com], a company active in the field of neurostimulation and biosensing, within which the neurostimulator presented in this work was designed.

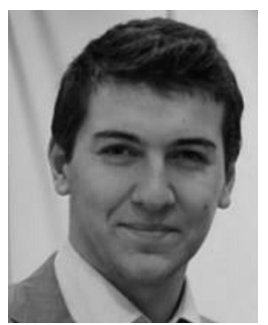

Yohan Botquin received his M.Sc. degree in Biomedical Engineering from Université Libre de Bruxelles, Belgium, in 2015. He then joined Synergia Medical where he is working as a R\&D engineer for the design of neurostimulators.

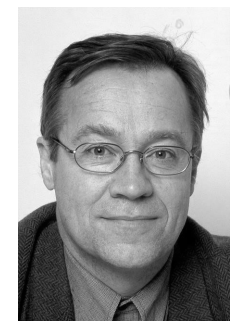

Bo Håkansson received his doctorate degree in 1984 at Chalmers University, Gothenburg, Sweden, regarding the bone-anchored hearing aid (BAHA). $\mathrm{He}$ has also initiated several new projects in bone conduction hearing such as a transcutaneous active hearing implant called the Bone Conduction Implant (BCI), a new audiometric bone conductor called B81 and bone conduction projects related to wireless communication. In recent years, Håkansson has been involved in projects for control of arm prostheses and projects to alleviate phantom pain.

The research has resulted in more than 100 scientific publications and 20 patent families. Håkansson is currently Professor of Biomedical Engineering at Chalmers University of Technology and director of the Biomedical Electrical Engineering research group.

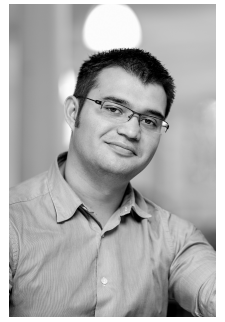

Max Ortiz Catalan (M'14) was born in Mexico in 1982. He received the Electronic Engineering degree from the ITESM Campus Toluca, Mexico, in 2005, the M.Sc. degree in Complex Adaptive Systems and the Ph.D. degree in Biomedical Engineering from Chalmers University of Technology (CTH), Gothenburg, Sweden, in 2009 and 2014, respectively. He is currently an Assist. Professor at $\mathrm{CTH}$ where he founded the Biomechatronics and Neurorehabilitation Laboratory (@ChalmersBNL). He works in close collaboration with the Centre for Advanced Reconstruction of Extremities (C.A.R.E.) at Sahlgrenska University Hospital, Gothenburg, Sweden. He also serves as Research Director for Integrum AB, Gothenburg, Sweden. He was recipient of the "You Can Make a Difference Award" in 2006, the "European Youth Award" in 2014, the "Delsys Prize" in 2016, and the "Brian \& Joyce Blatchford Award" in 2017.

His research interests include bioelectric signals acquisition electronics (analog and digital); signal processing and artificial intelligence algorithms for pattern recognition and control; neuromuscular interfaces; bone-anchored prostheses and osseointegration; as well as virtual and augmented reality for neuromuscular rehabilitation and the treatment of phantom limb pain. 